\title{
The parsec-scale structure of radio-loud broad absorption line quasars ${ }^{\star}$
}

\author{
G. Bruni ${ }^{1}$, D. Dallacasa ${ }^{1,2}$, K.-H. Mack ${ }^{1}$, F. M. Montenegro-Montes ${ }^{3}$, J. I. González-Serrano ${ }^{4}$, \\ J. Holt ${ }^{5}$, and F. Jiménez-Luján ${ }^{6,7}$ \\ 1 INAF-Istituto di Radioastronomia, via Piero Gobetti, 101, 40129 Bologna, Italy \\ e-mail: bruni@ira.inaf.it \\ 2 Università di Bologna, Dip. di Astronomia, via Ranzani, 1, 40129 Bologna, Italy \\ 3 European Southern Observatory, Alonso de Córdova 3107, Vitacura, Casilla 19001, Santiago, Chile \\ ${ }^{4}$ Instituto de Física de Cantabria (CSIC-Universidad de Cantabria), Avda. de los Castros s/n, 39005 Santander, Spain \\ 5 Leiden Observatory, Leiden University, PO Box 9513, 2300 RA Leiden, The Netherlands \\ ${ }^{6}$ Dpto. de Física Moderna, Universidad de Cantabria, Avda de los Castros s/n, 39005 Santander, Spain \\ 7 Centro Astronónico Hispano Alemán de Calar Alto (CAHA), C/ Jesús Durbán Remón 2-2, 04004 Almería, Spain
}

Received 21 February 2013 / Accepted 9 April 2013

\begin{abstract}
Context. Broad absorption line quasars (BAL QSOs) belong to a class of objects not well-understood as yet. Their UV spectra show BALs in the blue wings of the UV resonance lines, owing to ionized gas with outflow velocities up to $0.2 \mathrm{c}$. They can have radio emission that is difficult to characterize and that needs to be studied at various wavelengths and resolutions.

Aims. We aim to study the pc-scale properties of their synchrotron emission and, in particular, to determine their core properties.

Methods. We performed observations in the Very Long Baseline Interferometry (VLBI) technique, using both the European VLBI Network (EVN) at $5 \mathrm{GHz}$, and the Very Long Baseline Array (VLBA) at 5 and $8.4 \mathrm{GHz}$ to map the pc-scale structure of the brightest radio-loud objects of our sample, allowing a proper morphological interpretation.

Results. A variety of morphologies have been found: 9 BAL QSOs on a total of 11 observed sources have a resolved structure. Core-jet, double, and symmetric objects are present, suggesting different orientations. In some cases the sources can be young GPS or CSS. The projected linear size of the sources, also considering observations from our previous work for the same objects, can vary from tens of pc to hundreds of kpc. In some cases, a diffuse emission can be supposed from the missing flux-density with respect to previous lower resolution observations. Finally, the magnetic field strength does not significantly differ from the values found in the literature for radio sources with similar sizes.

Conclusions. These results are not easily interpreted with the youth scenario for BAL QSOs, in which they are generally compact objects still expelling a dust cocoon. The variety of orientations, morphologies, and extensions found are presumably related to different possible angles for the BAL producing outflows, with respect to the jet axis. Moreover, the phenomenon could be present in various phases of the QSO evolution.
\end{abstract}

Key words. quasars: absorption lines - galaxies: active - galaxies: evolution - radio continuum: galaxies

\section{Introduction}

The nature and origin of broad absorption line quasars (BAL QSOs) is still an open issue in the framework of active galactic nuclei (AGNs) morphology and evolution. The key characteristic of this class of objects ( $\sim 20 \%$ of the entire quasi stellar object (QSO) population, Hewett \& Foltz 2003) resides in their UV spectra, where broad absorption lines (BALs) are present in the blue wings of the UV resonance lines, owing to ionized gas with outflow velocities up to $0.2 \mathrm{c}$. Some evidence of BALs in young or luminous IR galaxies has led to proposing an evolutionary scenario (Briggs et al. 1984; Sanders 2002), in which this phenomenon is due to the young age of these objects: the central AGN would still be expelling the enveloping dust cocoon. The fraction of the QSOs showing BALs can then be interpreted as the duration of this phase in relation to the total active life. This view was supported by the presence of prototypical characteristics of young radio sources, like gigaHertz

* Table 3 is available in electronic form at http://www . aanda.org peaked sources (GPS) or compact steep sources (CSS), among BAL QSOs (Montenegro-Montes et al. 2008a; Liu et al. 2008).

An orientation model proposed by Elvis (2000) predicts that BAL outflows are present in all QSOs, but only when they intercept the line of sight would the BALs be detected. In this case the percentage of BAL QSOs would constrain the solid angle of the outflow. The bipolar wind model proposed by Punsly (1999a,b) was used by Ghosh \& Punsly (2007) to explain the polar BAL QSOs found by Zhou et al. (2006): in this model the BAL outflow would be aligned with the polar axis, while the relativistic jet would be nested inside of it. Recent works, based on large samples, do not confirm a young age for all BAL QSOs, but also a variety of orientations have been found (Bruni et al. 2012; DiPompeo et al. 2011). Also, on the arcsec-scale the majority of these sources remain unresolved in the radio band, with only about $10 \%$ or less showing an extended morphology (Bruni et al. 2012; DiPompeo et al. 2011).

Radio-loud BAL QSOs are a small fraction of the BAL QSO population $(\sim 10 \%$, Hewett \& Foltz 2003; Shankar et al. 2008), but the radio emission can be an additional tool for 
Table 1. Sample of the 11 radio-loud BAL QSOs studied in this paper.

\begin{tabular}{|c|c|c|c|c|c|c|}
\hline Name & SDSS name & $\begin{array}{c}\text { RA } \\
(\mathrm{J} 2000) \\
(3)\end{array}$ & $\begin{array}{c}\text { Dec } \\
(\mathrm{J} 2000) \\
(4)\end{array}$ & (5) & $\begin{array}{c}S_{1.4} \\
\text { (mJy/beam) } \\
(6)\end{array}$ & $\begin{array}{c}\text { BAL } \\
\text { Type } \\
\text { (7) }\end{array}$ \\
\hline $0044+00$ & J004444.06+001303.5 & 004444.0657 & +001303.560 & 2.28 & 53.1 & - \\
\hline $0756+37$ & J075628.25+371455.6 & 075628.24 & +371455.6 & 2.51 & 239.4 & - \\
\hline $0816+48$ & J081618.99+482328.4 & 081618 . & +482328.469 & 3.57 & 68.3 & - \\
\hline $0849+27$ & J084914.27+275729.7 & 084914.2 & +275729.709 & 1.73 & 52.8 & $\mathrm{Hi}$ \\
\hline $1014+05$ & $\mathrm{~J} 101440.35+053712.6$ & 101440.3561 & +053712.682 & 2.01 & 55.0 & $\mathrm{Hi}$ \\
\hline $1102+11$ & J110206.66+112104.9 & 110206.6617 & +112104.900 & 2.35 & 82.3 & - \\
\hline $1237+47$ & $\mathrm{~J} 123717.44+470807.0$ & 123717.44 & +470807.0 & 2.27 & 78.5 & FeLo \\
\hline $1304+13$ & $\mathrm{~J} 130448.06+130416.6$ & 130448.0594 & +130416.590 & 2.57 & 49.6 & - \\
\hline $1327+03$ & $\mathrm{~J} 132703.21+031311.2$ & 132703.21 & +031311.2 & 2.83 & 60.7 & - \\
\hline $1406+34$ & $\mathrm{~J} 140653.84+343337.3$ & 140653.84 & +3433 37.3 & 2.56 & 164.4 & - \\
\hline $1603+30$ & $\mathrm{~J} 160354.15+300208.6$ & 160354.1534 & +300208.705 & 2.03 & 53.7 & $\mathrm{Hi}$ \\
\hline
\end{tabular}

Notes. Columns 3-5 are respectively RA, Dec and redshift as measured from the SDSS. For phase-referenced sources we give the coordinates of the brightest component peak, derived from our VLBI observations. Column 6 gives the peak flux density at $1.4 \mathrm{GHz}$ from the FIRST catalogue (Becker et al. 1995; White et al. 1997). The last column is the BAL type as given in Bruni et al. (2012): a hyphen indicates that it could not be determined.

understanding the orientation and the age (O'Dea \& Baum 1997) of these sources. We focus on this class of objects to collect information that can help in understanding which of the two models is the most probable. Thus, we embarked on a VLBI project to test the inner structure of the QSO.

The VLBI technique is an important tool for this kind of study, allowing one to reach the necessary resolution and sensitivity to study distant objects like BAL QSOs. This approach offers different indicators of the morphology of the radio source: (1) The detection of radio jets and their asymmetry can provide constraints on the radio source orientation; (2) The spectral index analysis allows us to discriminate between core-jet or double structure; (3) Variability with respect to the total flux-density already measured with the VLA can give an estimate of the activity and orientation of the QSO jet axis with respect to the line of sight. (4) In case the pc-scale structure displays mini-lobes, which are typically found in young radio sources, the projected linear size of the radio source can be used to estimate the kinematic age (Dallacasa et al. 2003).

In previous works on BAL QSOs, using the VLBI imaging technique (Jiang \& Wang 2003; Kunert-Bajraszewska et al. 2007, 2010; Liu et al. 2008; Montenegro-Montes et al. 2008b; Gawroński \& Kunert-Bajraszewska 2011), various morphologies and sizes have been found, and in most cases sources were unresolved even at pc-scale resolution. In this paper we present the first results of an extensive observational campaign to determine the pc-scale structure and morphology of a complete sample of radio-loud BAL QSOs, whose characteristics in the radioband were presented in Bruni et al. (2012). Beyond the study of the pc-scale radio properties of the BAL QSO population, we aim at distinguishing which of the presented models could be the most suitable for explaining the BAL phenomenon.

This paper is organized as follows. In Sect. 2 we present the sources and the observations, and in Sect. 3 we show the pc-scale radio maps and discuss the morphology. In Sect. 4 we discuss our results in the light of earlier works in the literature.

The cosmology adopted throughout the paper assumes a flat universe and the following parameters: $H_{0}=70 \mathrm{~km} \mathrm{~s}^{-1} \mathrm{Mpc}^{-1}$, $\Omega_{\Lambda}=0.7, \Omega_{\mathrm{M}}=0.3$. The adopted convention for the spectral index definition is $S_{v} \propto v^{\alpha}$.

\section{Radio observations}

This work is based on a well-defined sample of radio-loud BAL QSOs presented in Bruni et al. (2012). This sample has been selected from the fourth release of the Sloan Digitized Sky Survey (SDSS) QSO catalogue (Schneider et al. 2007), with a fluxdensity threshold of $30 \mathrm{mJy}$ at $1.4 \mathrm{GHz}$ and a redshift range of $1.7<z<4.7$ : these criteria resulted in a sample of $25 \mathrm{ob}-$ jects. The radio spectra of the sources, presented in Bruni et al. (2012), show that BAL QSOs are weaker at radio-frequencies above $1.4 \mathrm{GHz}$, with a few exceptions, requiring sensitive observations to study their pc-scale structure.

In this paper, we present VLBI observations of 11 of the brighter sources $\left(S_{1.4}>\sim 50 \mathrm{mJy}\right)$ included in our sample. These 11 are a random subset of these brighter sources. The high-resolution study of sources $1159+01\left(S_{1.4}=266 \mathrm{mJy}\right)$ and $1624+37\left(S_{1.4}=56 \mathrm{mJy}\right)$, not included here, has already been presented by Montenegro-Montes et al. (2008b): we thus obtained parsec-scale radio maps for a total of 13 out of 25 BAL QSOs in the whole sample. The study of the polarized flux density was not possible in these works, since much more observing time would have been needed to detect the low fraction of polarized flux density of these objects. More information on overall radio spectra and the kpc-scale polarization properties of the objects studied here, as well as the remaining sources in the sample, are available in Bruni et al. (2012). In Table 1 we report some basic information on the 11 BAL QSOs presented here, while a summary of the observations is reported in Table 2.

All the data reduction and map analysis was performed using the NRAO AIPS ${ }^{1}$ software, following the usual procedures once editing of raw visibilities and of antenna temperature (TSYS) measurements were carried out. Based on the variation in the amplitude solutions once system temperatures and antenna gain have been considered, the accuracy of the flux-density scale can be estimated to be within $\pm 5 \%$. The final angular resolution of the maps is in the range $2-5$ mas.

\subsection{VLBA data}

Seven objects were observed with the Very Long Baseline Array (VLBA) at 5 and $8.4 \mathrm{GHz}$ (C and X-band, respectively) with a

1 http://www.aips.nrao.edu 
G. Bruni et al.: The parsec-scale structure of radio-loud broad absorption line quasars
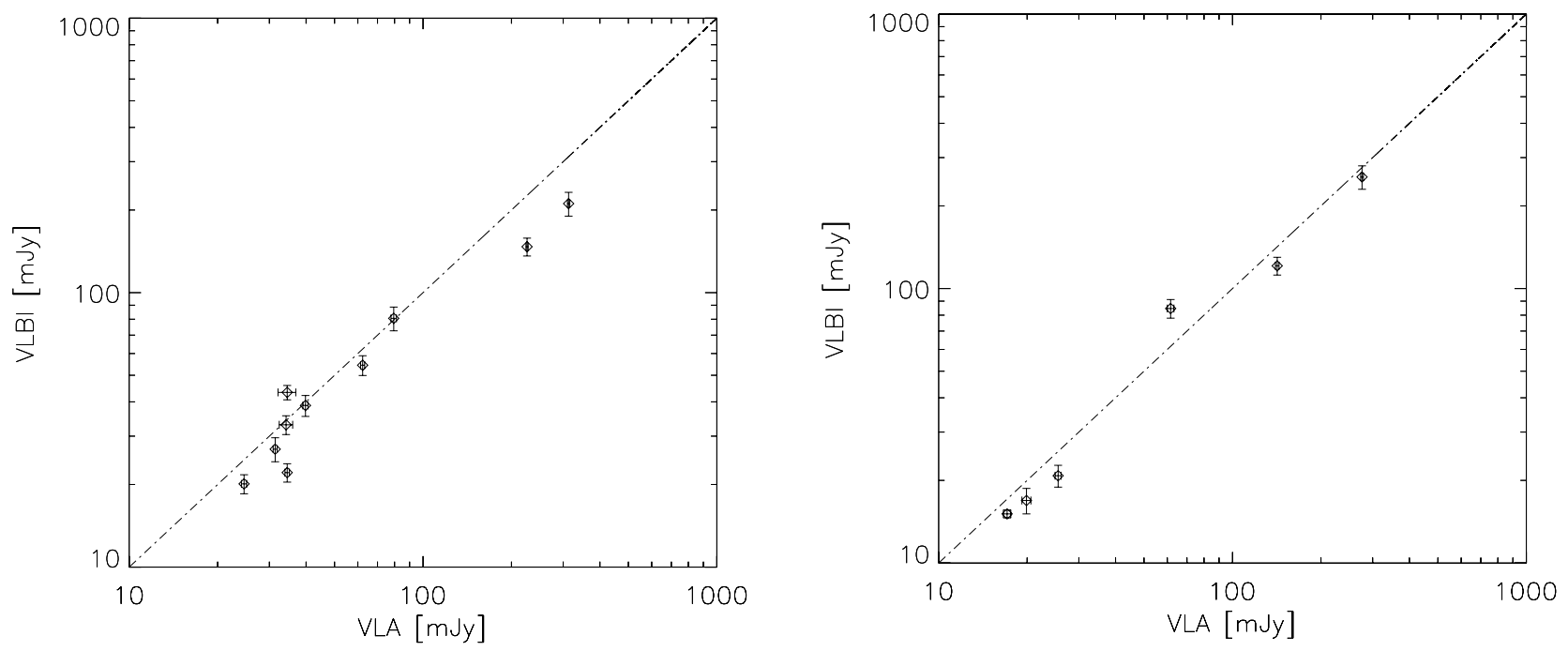

Fig. 1. Flux densities of VLBI vs VLA at 5 (left) and $8 \mathrm{GHz}$ (right), for all of the sources with both measurements. The dashed line indicates a slope equal to 1 . The outliers $\left(\sigma_{\mathrm{var}}>3\right)$ are sources $0756+37,1406+34$ and $1014+05$ at $5 \mathrm{GHz}$, and $1237+47$ at $8.4 \mathrm{GHz}$.

Table 2. Summary of the observations and instrumental setup.

\begin{tabular}{ccccccc}
\hline \hline Run & \multicolumn{1}{c}{ Date } & Telescope & $\begin{array}{c}\text { Frequencies } \\
(\mathrm{GHz})\end{array}$ & $\begin{array}{c}\text { Bandwidth } \\
(\mathrm{MHz})\end{array}$ & $\begin{array}{c}\theta_{\mathrm{HPBW}} \\
(\mathrm{mas})\end{array}$ & $\begin{array}{c}\text { Time on source } \\
(\mathrm{h})\end{array}$ \\
\hline 1 & 25-26 Oct. 2009 & EVN & 5 & 32 & 5 & 2 \\
2 & 19, 22, 26, 28 Feb. 2010 & VLBA & $5,8.4$ & 64 & 4,2 & 1,2 \\
3 & 4, 7, 23, 26 Apr.-1, 2 May 2011 & VLBA & $5,8.4$ & 64 & 4,2 & 3,4 \\
\hline
\end{tabular}

Notes. In the penultimate column the typical beam sizes are given (half-power beam-width).

total bandwidth of $64 \mathrm{MHz}$ at each frequency, in two observing runs in 2010 and 2011. In the first run an average on-source time of about one hour at $5 \mathrm{GHz}$ and two hours at $8.4 \mathrm{GHz}$ was allowed. The correlation was performed with the VLBA correlator at the National Radio Astronomy Observatory (NRAO) in Socorro (US). In the second run, when the fainter sources were observed, a total of three hours at $5 \mathrm{GHz}$ and four hours at $8.4 \mathrm{GHz}$ were spent on each target. The data were then processed with the new Distributed FX (DiFX) software correlator (Deller et al. 2011). Given that, in general, the target BAL QSOs were weak; the VLBA observations were carried out in phase referencing mode for most of sources; the transfer of delay, phase, and phase rate solutions from a reference source close to target allowed absolute positions to be obtained, which significantly improved the earlier information taken from the FIRST catalogue (Becker et al. 1995; White et al. 1997). Therefore, the phase centre in the maps presented in Fig. 3 refers to the shift applied and not to the peak in the map plane whose position is instead reported in Table 1 . Since the correlation was done using the position from the FIRST survey, we had to allow a rather wide field for the initial imaging of each target source. In fact we started with about $1^{\prime \prime}$ wide maps, and once the target source was detected, a smaller field was selected after applying appropriate shifts in RA and DEC in order to have the target source at about the centre of the map. The same shift was applied at both frequencies. Then a few iterations of phase self-calibration were performed. The final maps are shown in Fig. 3 and are discussed in the next section.

\subsection{EVN data}

Four additional sources were observed with the European VLBI Network (EVN) at $5 \mathrm{GHz}(C$-band), in 2009, using the antennas in Jodrell Bank, Westerbork, Effelsberg, Onsala, Medicina,
Torun, Shanghai, Urumqi, Noto, Yebes, and the two MERLIN telescopes in Darnhal and Knockin. A total bandwidth of $32 \mathrm{MHz}$ and an average on-source time of about two hours were allowed. The data were correlated at the Joint Institute for VLBI in Europe (JIVE) correlator in Dwingeloo (The Netherlands).

For three of the sources we carried out phase-referencing. The maps thus adopt the same convention as for the VLBA ones. Standard procedures, including fringe-fitting, were used for the data reduction of the target sources. Delay, phase, and rate solutions were generally found above the standard signal-to-noise ratio threshold, for all the target sources.

\section{Results}

Once final maps had been obtained, we carried out a 2D Gaussian fitting solving for component position, fluxdensity, size, and position angle. The resulting information is reported in Table 3. The distance between the centroids of each individual component as found by the fits were used to calculate the total projected angular and linear sizes of the source.

In the following, we briefly describe each source, observed with the VLBA (Sect. 3.1) or the EVN (Sect. 3.2). For the often mentioned overall radio-spectrum of the sources we refer to Bruni et al. (2012). Figure 1 provides a comparison with the VLA flux-densities presented in our previous work. In this section we also discuss any hint of variability for the individual sources. Finally, in Table 1 we provide the position of the brightest component peak for the seven sources with absolute position information from phase-referencing.

\subsection{VLBA maps}

In this section, the pc-scale structure of the BAL QSOs observed with the VLBA is briefly discussed. Most of the sources have 
A\&A 554, A94 (2013)
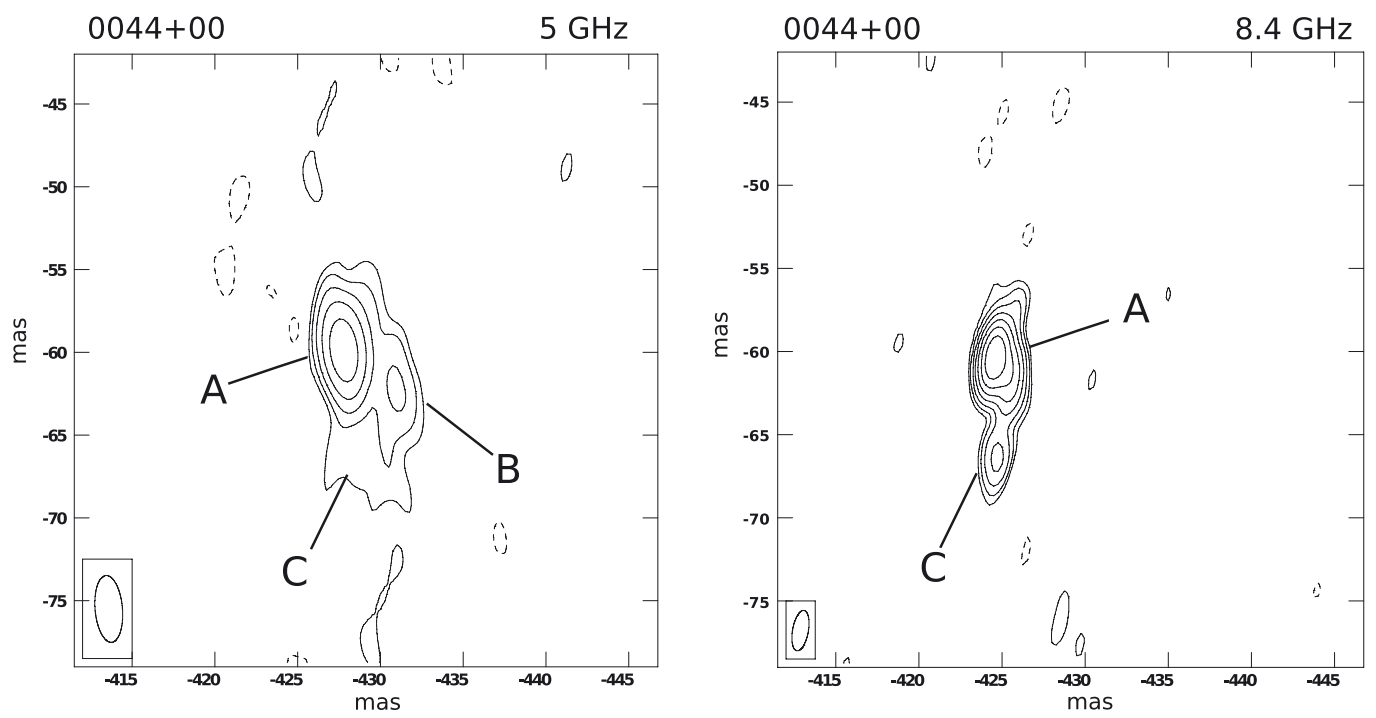

Levels $=0.7 *(-1,1,2,4,8,16,32,64) \mathrm{mJy} /$ beam

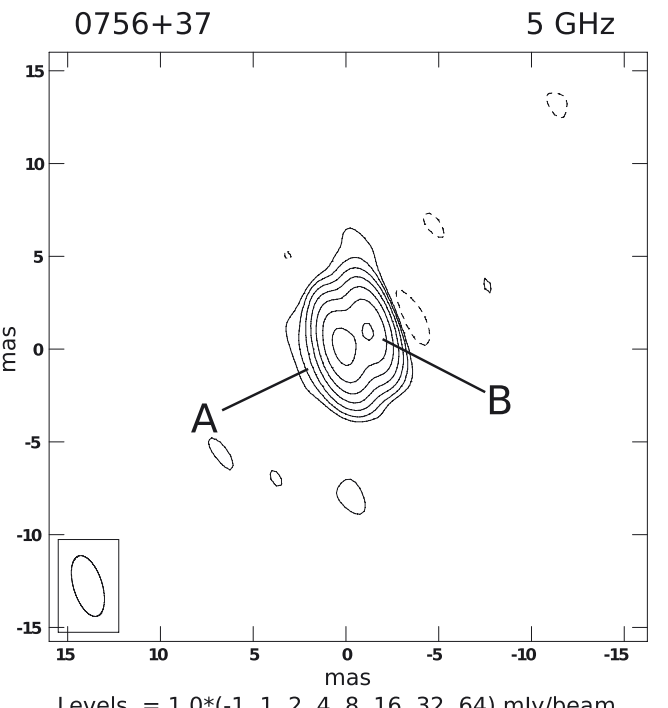

Levels $=0.1 *(-1,1,2,4,8,16,32,64) \mathrm{mJ} / \mathrm{beam}$

$0756+37$

$8.4 \mathrm{GHz}$

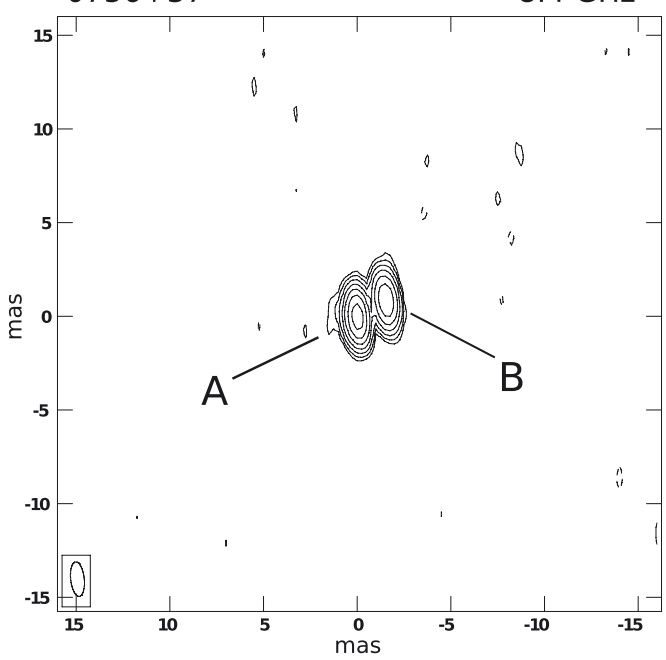

Levels $=0.7 *(-1,1,2,4,8,16,32,64) \mathrm{mJy} / \mathrm{beam}$
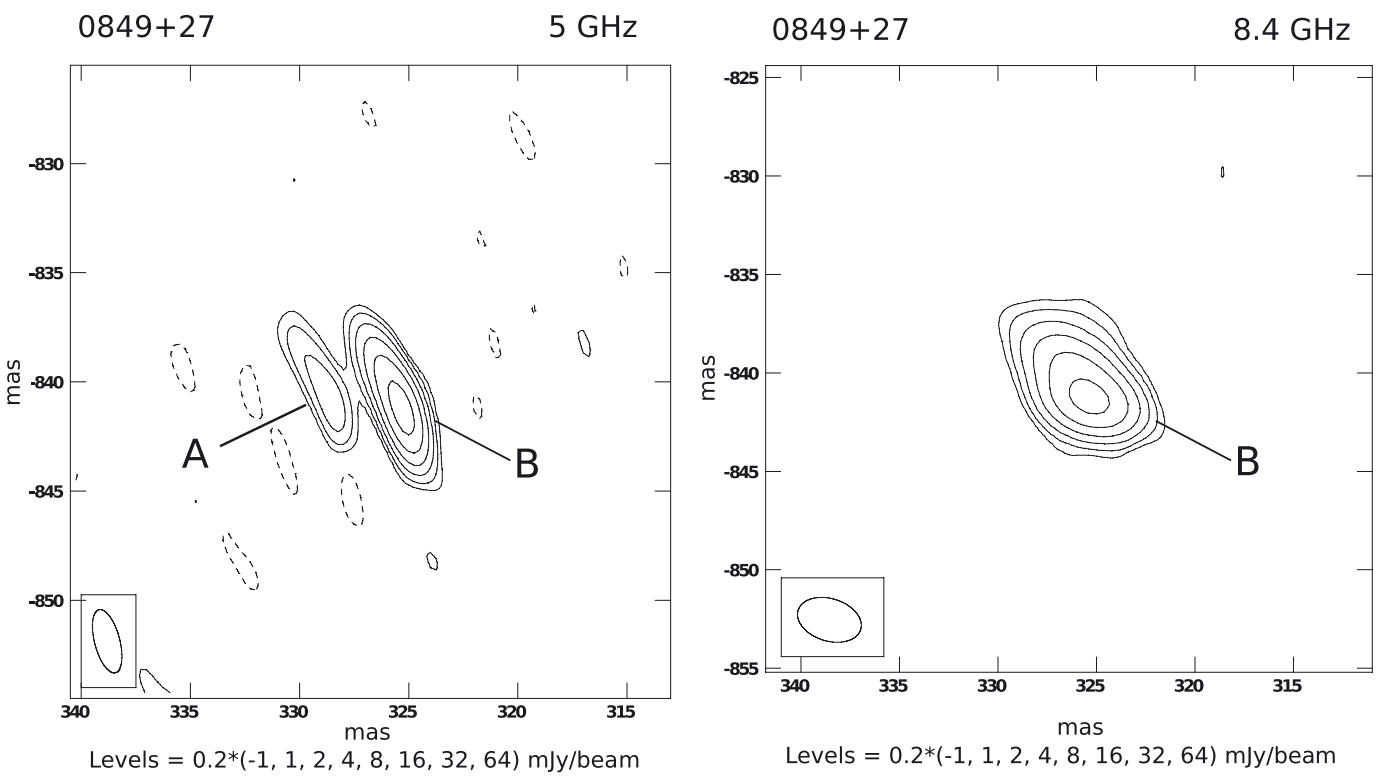

Fig. 2. Maps of the 6 BAL QSOs resolved with the VLBA. The synthesized beam size is shown in the lower left corner of the map. Levels are $3 \sigma$ multiples, according to the legend. Orientation is N-up, E-left. 
G. Bruni et al.: The parsec-scale structure of radio-loud broad absorption line quasars
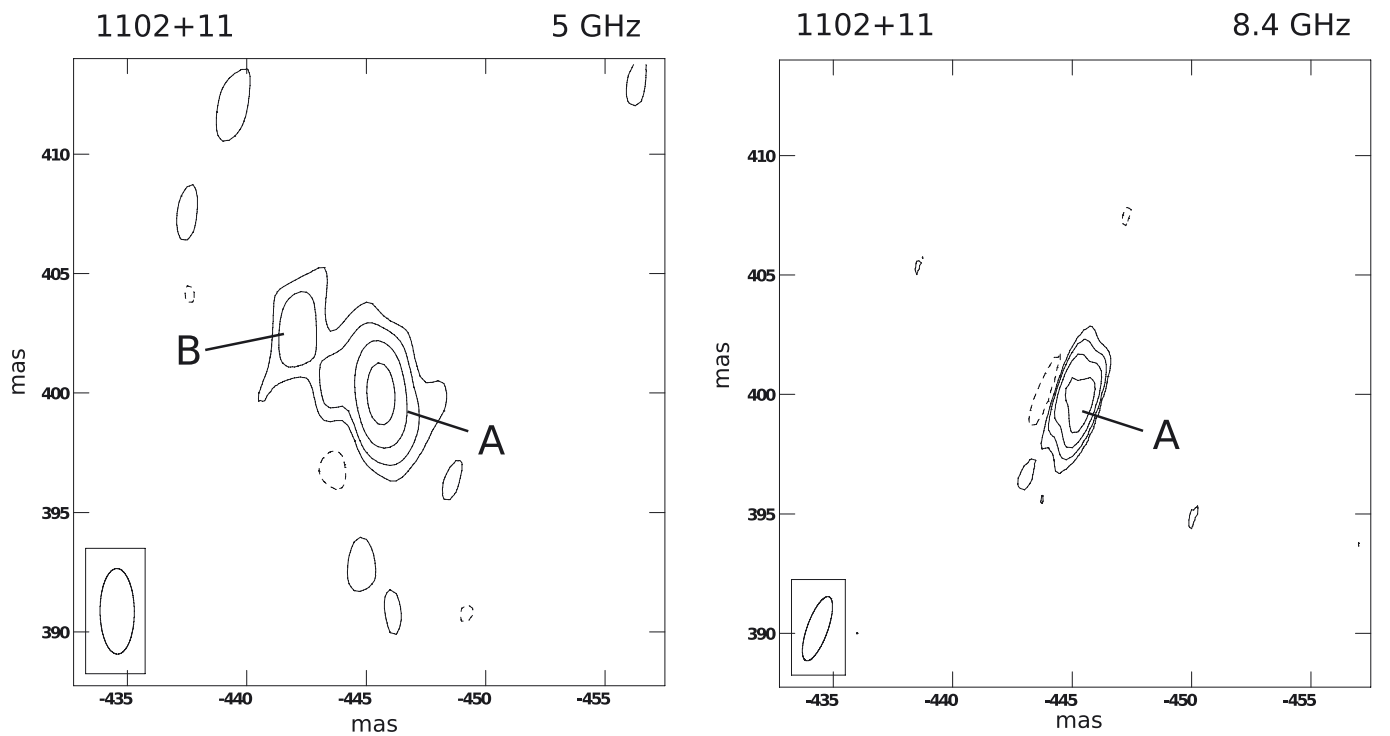

Levels $=1.8 *(-1,1,2,4,8,16,32,64) \mathrm{mJy} / \mathrm{beam}$ Levels $=0.9 *(-1,1,2,4,8,16,32,64) \mathrm{mJy} / \mathrm{beam}$
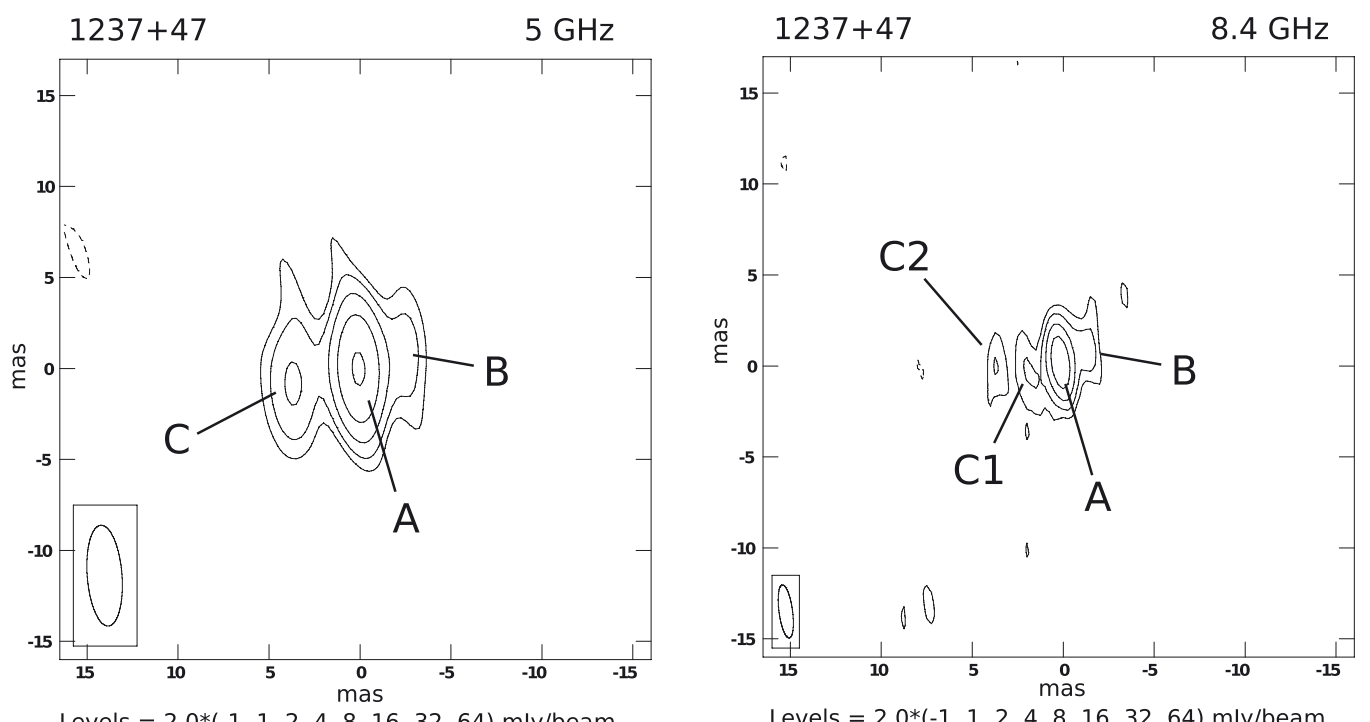

Levels $=2.0 *(-1,1,2,4,8,16,32,64) \mathrm{mJy} / \mathrm{beam}$
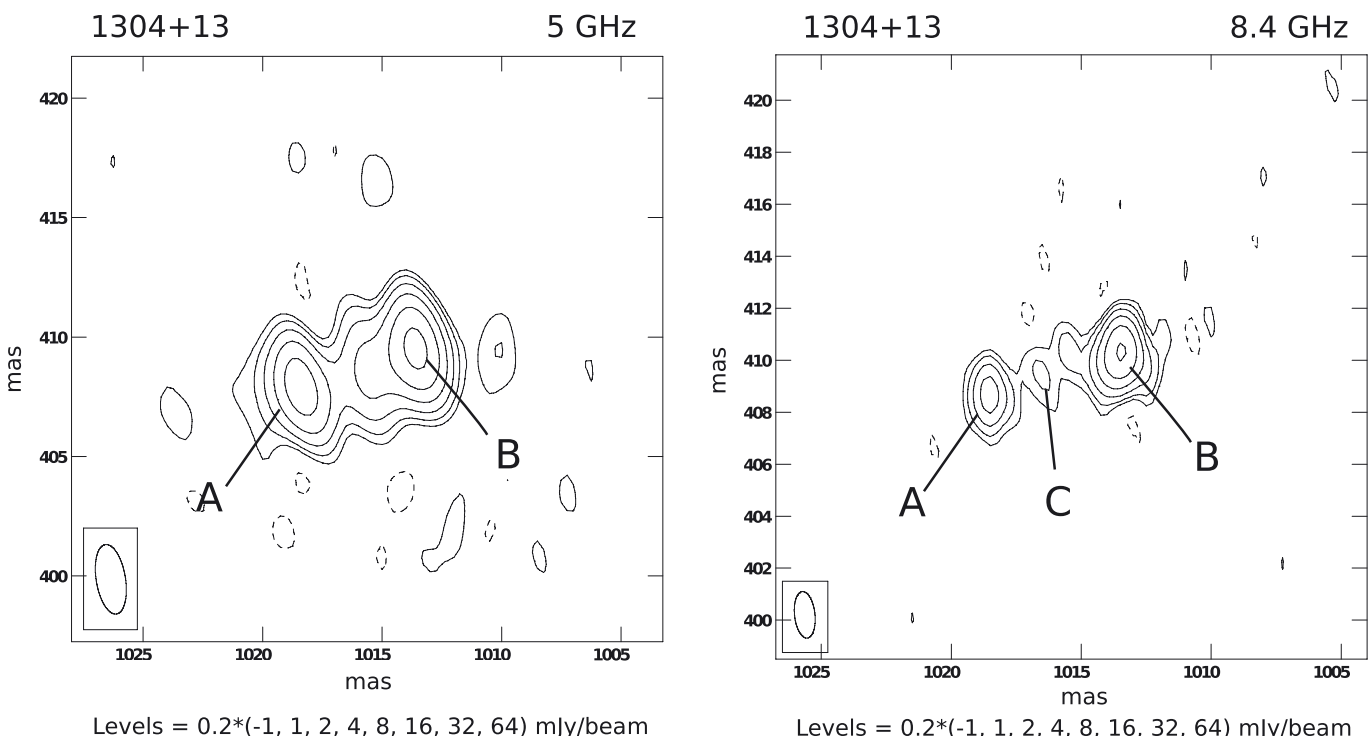

Levels $=0.2 *(-1,1,2,4,8,16,32,64) \mathrm{mJ} / \mathrm{beam}$

Fig. 2. continued. 
been found to be unresolved in the earlier arcsecond-scale observations. The typical resolution of the VLBA maps is $\sim 4$ mas at $5 \mathrm{GHz}$ and $\sim 2$ mas at $8.4 \mathrm{GHz}$. The spectral indices $\alpha_{5}^{8.4}$ for the individual components were calculated using the integrated flux density from the Gaussian fit, and do not refer to any local values. The availability of maps at two frequencies often helps in correctly interpreting the source morphology in terms of cores or steep spectrum components.

\section{$0044+00$}

Both maps at $5 \mathrm{GHz}$ and $8.4 \mathrm{GHz}$ show a resolved structure. In particular at $5 \mathrm{GHz}$, three misaligned components are present: two of them are clearly visible, the third is embedded in some diffuse structure. While a Gaussian fit was possible for components $\mathrm{A}$ and $\mathrm{B}$, for component $\mathrm{C}$ it did not converge since it barely stands against some diffuse emission, and only an upper limit for its flux-density could be obtained. In case the map at $8.4 \mathrm{GHz}$ was not available, component $\mathrm{C}$ would not have been considered. In this $8.4 \mathrm{GHz}$ map, much deeper than that at $5 \mathrm{GHz}$, only components $\mathrm{A}$ and $\mathrm{C}$ are visible, suggesting a steep spectral index for component B whose surface brightness drops below the detection level. Component $\mathrm{A}$ has a rather flat spectral index $(-0.52 \pm 0.27)$ and could be most probably classified as the core region, in which part of the jet is also visible, as suggested by the elongation in $\mathrm{PA} \sim 176^{\circ}$.

Although it is not straightforward, the morphology of this source can be interpreted as a core-jet.

\section{$0756+37$}

In both VLBA bands we resolve the radio source into two components, separated by $\sim 1.8$ mas $(\sim 15 \mathrm{pc}$ at the redshift of the object) at the highest resolution, in PA $\sim-60^{\circ}$. Both components have similar sizes and spectral indexes $(-0.38 \pm 0.27$ for $\mathrm{A}$ and $-0.37 \pm 0.27$ for B). Since the overall spectrum peaks at $2.5 \mathrm{GHz}(8.7 \mathrm{GHz}$ rest frame), and it is steep in the optically thin (power-law, not self-absorbed) part of the emission, we can infer that we have detected two hot spots in the mini-lobes of a typically young radio source.

The VLBA can account for a fraction of the total flux density measured in earlier VLA observations $(\sim 65 \%$ at $5 \mathrm{GHz}$ and $\sim 85 \%$ at $8.4 \mathrm{GHz}$ ): considering the variability significance as defined by Zhou et al. (2006), the discrepancy is significant at $5 \mathrm{GHz}$, with a $\sigma_{\text {var }}=7.0$, while at $8 \mathrm{GHz}$ we obtain $\sigma_{\text {var }}<3$. The time interval between VLA and VLBA observations is about seven months. Thus, either the source is variable - but this would be inconsistent with the hot-spot scenario - or, more likely, there is some emission on angular scales not sampled by the VLBA (tens of mas or more).

This source was observed also by Gawroński \& Kunert-Bajraszewska (2011) using the EVN at $1.6 \mathrm{GHz}$, and reporting a probable core-jet structure, but no maps were provided in that work.

\section{$0849+27$}

This source has already been resolved in the FIRST survey (VLA at $1.4 \mathrm{GHz}$, see Bruni et al. 2012) showing three components with a projected linear size of $382 \mathrm{kpc}$, and a position angle for the structure of $\sim 45^{\circ}$. These VLBA observations were designed to study the central component, which was unresolved on the arcsecond scale. The maps show two components at $5 \mathrm{GHz}$ (A and B) separated by 3.5 mas (30 pc), while only B is visible at $8.4 \mathrm{GHz}$, with a spectral index of $-0.12 \pm 0.20$ (flat) that can be considered as the source core. This component is resolved at both frequencies in $\mathrm{PA} \sim 30^{\circ}$.

\section{$1102+11$}

The map at $5 \mathrm{GHz}$ shows a structure resolved into two components, which are separated by $\sim 4.1$ mas $(\sim 34 \mathrm{pc})$, in PA $-53^{\circ}$. At $8.4 \mathrm{GHz}$ only the strongest component (A) has been detected, and it turned out to be resolved along the same direction. Its spectral index is $-0.97 \pm 0.29$. Such a steep spectrum makes it unlikely that component $\mathrm{A}$ is the source core. It is also too steep to be an active hot spot. Component $\mathrm{B}$ has probably an even steeper spectral index, since the flux density is below the $3 \sigma$ significance limit at $8.4 \mathrm{GHz}$. All this makes the morphological classification of the pc-scale structure rather unclear.

The source presents a convex radio spectrum, although more observations at low frequencies are needed to clearly determine the turnover frequency, if present.

\section{$1237+47$}

This source is resolved into three components at $5 \mathrm{GHz}$, with the strongest one in the centre (A). At $8.4 \mathrm{GHz}$, the eastern component $\mathrm{C}$ is resolved in two subcomponents $(\mathrm{C} 1$ and $\mathrm{C} 2)$, both aligned with $\mathrm{A}$ and $\mathrm{B}$. Component $\mathrm{A}$ has an inverted spectral index $(0.41 \pm 0.29)$ and thus could be interpreted as the core, while $\mathrm{B}$ and $\mathrm{C} 1$ present elongated emission toward $\mathrm{A}$, with a similar flux density at $8.4 \mathrm{GHz}$. It could be the inner part of the jets, with an additional hot spot $(\mathrm{C} 2)$ visible in the direction of $\mathrm{C} 1$. The projected linear size from $X$ band is 4.8 mas (40 pc).

From our previous radio observations of this source we know that it presents a flat spectrum, which can possibly be approximated with a convex shape with a peak at about $3.5 \mathrm{GHz}$. Thus, this object can be a young radio source in which the core region is still the brightest in the source. Moreover, at $8.4 \mathrm{GHz}$ its flux density exceeds $(\sim 37 \%)$ the total flux density in our earlier VLA observation. This could indicate that there is still a very active phase in which new components are likely to be formed on short timescales, creating the variability in the flux density. The time interval between VLA and VLBA observations is about seven months, with a $\sigma_{\text {var }}=3.4$, just above the $3-\sigma$ significance threshold.

\section{$1304+13$}

It was possible to resolve this source both at $5 \mathrm{GHz}$ and at $8.4 \mathrm{GHz}$ : in the maps, the two brightest components are on the edges of the radio source ( $\mathrm{A}$ and $\mathrm{B}$ ), while the central component $\mathrm{C}$ is distinguishable only at $8.4 \mathrm{GHz}$. The fit did not converge for this last component, so we could only estimate the flux density upper limit.

The total projected linear size of the source at $8.4 \mathrm{GHz}$ is 5.3 mas (44 pc). The overall spectrum is steep, and does not present a peak in the $\mathrm{GHz}$ range, which is the only one that could be investigated at the moment.

\section{$1406+34$}

This source is unresolved in both bands with the VLBA. We can set an upper limit of 1.8 mas $(14 \mathrm{pc})$ to its size. The map at $8.4 \mathrm{GHz}$ shows a noise pattern that is not related to any extended 


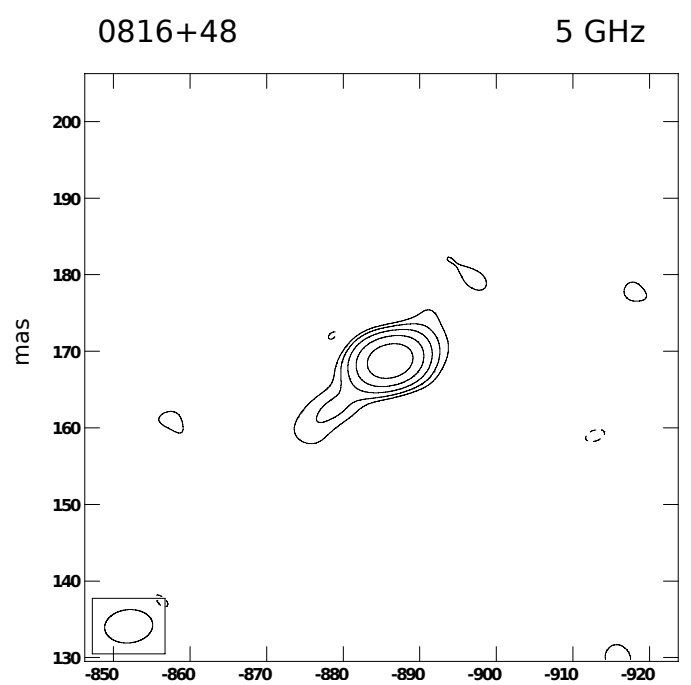

Levels $=0.8^{*}(-1,1,2,4,8,16,32,64) \mathrm{mJy} / \mathrm{beam}$ $1014+05$

$5 \mathrm{GHz}$

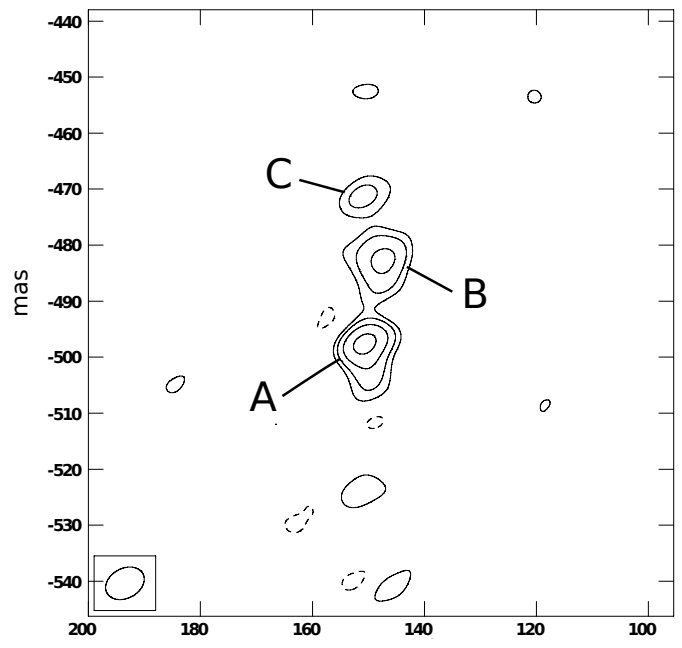

Levels $=0.9 *(-1,1,2,4,8,16,32,64) \mathrm{mJy} /$ beam

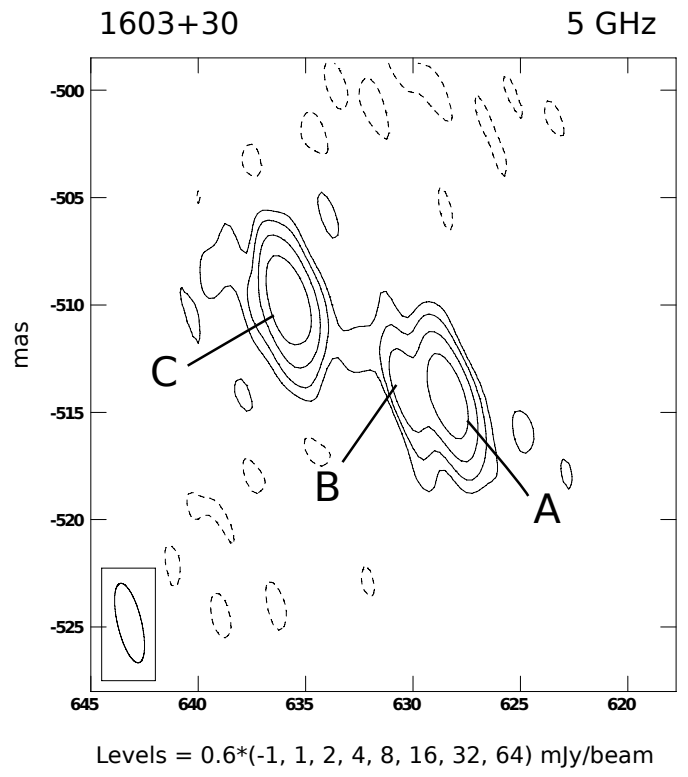

Fig. 3. Maps of the 3 BAL QSOs resolved with the EVN. The synthesized beam size is shown in the lower left corner of the map. Levels are $3 \sigma$ multiples, according to the legend. Orientation is N-up, E-left. structure. The pc-scale emission revealed by the present observations has an inverted spectrum $(\alpha=0.36 \pm 0.26)$, and we are therefore seeing the optically thick, self-absorbed part of the synchrotron emission.

The overall radio spectrum peaks at about $5.4 \mathrm{GHz}$. The VLBA flux-density measurements at $5 \mathrm{GHz}$ accounts only for $\sim 67 \%$ that of the VLA $\left(\sigma_{\mathrm{var}}=4.8\right)$, owing either to some intrinsic variability or to an additional component fully resolved on the pc-scale. Such a component, with a steep spectrum, may be responsible for the $327 \mathrm{MHz}$ emission detected in the WENSS survey (de Bruyn et al. 2000), which could be the remnant of an earlier episode of radio activity and which also modifies the spectral slope at low frequencies. The source is also unresolved in VLA maps.

\subsection{Results from EVN observations}

The EVN observed four BAL QSOs at $5 \mathrm{GHz}$ ( $C$-band). This array is made of various radio telescopes with substantially different performances. In particular, the presence of a large dish like the Effelsberg-100 m allows better baseline sensitivity than on a typical VLBA baseline. However, due to the geographical distribution of the telescopes, the uv-plane is not uniformly sampled, and, on average, the structural information that can be derived here is poorer than the maps presented in the previous section. The typical resolution of these maps is $\sim 5$ mas.

\section{$0816+48$}

This source shows an elongation toward SE. From the major axis of the Gaussian fit (6.8 mas) we can infer an upper limit of projected linear size of $51 \mathrm{pc}$. A clear classification of the spectrum was not possible in our previous work, but the VLA map at $1.4 \mathrm{GHz}$ already showed an elongation toward SW, not corresponding with the one present here, and with a major and minor axes of 29 and 14 arcsec, corresponding to 217 and $105 \mathrm{kpc}$, respectively. This could be another case of intermittent activity with a change in the jet orientation between the two phases. Further observations at the intermediate VLA resolution could confirm this hypothesis.

\section{$1014+05$}

This source presents a complex morphology whose proper classification is rather difficult, because the brightest component (A) can be interpreted as the core region, and there are two more components (B and C) toward north. The overall structure is well aligned in $\mathrm{PA} \sim 0^{\circ}$.

The distance between the centroids of $\mathrm{A}$ and $\mathrm{C}$ components is $\sim 26.6$ mas, corresponding to a projected linear size of 229 pc. In our earlier work we found a power-law spectrum for this source, but more data below $1.4 \mathrm{GHz}$ are needed to exclude any turnover in the $\mathrm{MHz}$ range.

Our EVN map can account for $\sim 59 \%$ of the total flux density measured in the VLA map $\left(\sigma_{\text {var }}=8.2\right)$, since this discrepancy may arise either from some flux-density variability or from an additional structure not sampled by the EVN spacings.

\section{$1327+03$}

This is an unresolved source. The upper limit for the linear size, estimated from the Gaussian fit, is $46 \mathrm{pc}(5.9 \mathrm{mas})$ and $11 \mathrm{pc}$ (1.4 mas), respectively, on the major and minor axes, but the 
beam is considerably elliptical in this map so this can only be an indicative value. A power-law spectrum down to $1.4 \mathrm{GHz}$ does not show any turnover for this source.

\section{$1603+30$}

In this case a rather symmetric structure can be seen on the parsec scale. The south-western component can be fitted with two (A and B) Gaussians. The separation between A and C (at the north-eastern edge) components is 8.6 mas, with a projected linear size of $74 \mathrm{pc}$.

This source presents a convex radio spectrum, with a peak frequency at $1.4 \mathrm{GHz}$, and thus could be classified as a young GPS source, in which the emission is dominated by the hot spots.

On the arcsecond scale, a VLA map at $22 \mathrm{GHz}$ shows a resolved structure in which some additional emission is present at about 2 arcsec $(17 \mathrm{kpc})$ to the south. Such a linear size is more typical of CSS sources (1-20 kpc), although the overall spectrum is typical of GPS sources. The structure seen with the EVN is not aligned with the one seen with the VLA: probably a merger or a jet precession could justify the different morphologies of the same object on different scales. This is another case of a possibly young source, like $1237+47$, with a flux density at VLBI resolution exceeding the earlier VLA observations at the same frequency $(\sim 25 \%)$, but in this case the variability significance $\sigma_{\text {var }}$ is only 2.5 , i.e. below the threshold. This source has already been observed with EVN+MERLIN at $1.6 \mathrm{GHz}$ (Liu et al. 2008), but owing to the lower resolution $(13.0 \times 4.0$ mas $)$ it was unresolved.

\subsection{Magnetic field estimation}

We applied the Govoni \& Feretti (2004) formulae to calculate the magnetic field intensity or strength B. Supposing the synchrotron mechanism to be at the origin of the radio emission, this estimate assumes that the magnetic-field energy density $U_{B}$ and the relativistic particles energy density $U_{\text {part }}$, contained in the emitting region, contribute with approximately equal values to the total energy density $U_{\text {tot }}$. This equipartition assumption allows minimizing $U_{\text {tot }}$, providing a conservative estimate of $\mathrm{B}$. The formulae for minimum energy density, $u_{\min }$, and the corresponding equipartition value for the magnetic field strength, $B_{\text {eq }}$, are the following:

$$
\begin{aligned}
& u_{\min }\left[\frac{\mathrm{erg}}{\mathrm{cm}^{3}}\right]= \xi\left(\alpha, v_{1}, v_{2}\right)(1+k)^{4 / 7}\left(v_{0[\mathrm{MHz}]}\right)^{4 \alpha / 7}(1+z)^{(12+4 \alpha) / 7} \\
&\left.\times\left(I_{0\left[\frac{\mathrm{m} J}{\operatorname{arsec}^{2}}\right]}\right]\right)^{4 / 7}(d[\mathrm{kpc}])^{-4 / 7} \\
& B_{\mathrm{eq}}=\left(\frac{24 \pi}{7} u_{\mathrm{min}}\right)^{1 / 2}
\end{aligned}
$$

where $z$ is the source redshift, $I_{0}$ the source brightness at the frequency $v_{0}, d$ the source depth, $k$ the proton-to-electron energy ratio, $v_{0}$ the observed frequency, and $\alpha$ the spectral index of the synchrotron emission (adopting the convention $S_{v} \propto v^{-\alpha}$ ). The constant $\xi\left(\alpha, v_{1}, v_{2}\right)$, depending on $\alpha$ and on the minimum and maximum energy of the charged particles (corresponding to $v_{1}$ and $v_{2}$ ), is tabulated in Table 1 of Govoni \& Feretti (2004). We chose a value of $2.5 \times 10^{-12}$ corresponding to $\alpha=0.5$, and minimum and maximum emission frequencies of $10 \mathrm{MHz}$ and $100 \mathrm{GHz}$, respectively. In this calculation we assumed $k=1$, a magnetic field filling-factor $\phi=1$, and an ellipsoidal volume for the component, with depth $d$ equal to the minor axis.
Assuming a low-energy cut-off in the particle energy distribution, the derived magnetic field $B_{\mathrm{eq}}^{\prime}$ is related to $B_{\mathrm{eq}}$ by the following formula (Brunetti et al. 1997):

$B_{\mathrm{eq}}^{\prime} \sim \gamma_{\min }^{\frac{1-2 \alpha}{3+\alpha}} B_{\mathrm{eq}}^{\frac{7}{2(3+\alpha)}}$.

We assumed a minimum Lorentz factor $\gamma_{\min }=100$ in this calculation.

In Table 3 we present the results of the magnetic field estimate for each component (or for the all the sources in case it is unresolved). For the unresolved sources or components, having deconvolved dimensions equal to zero, we used the major and minor axes of the beam and the peak flux density. Components without Gaussian fit were omitted. For two sources $(1237+47$ and $1406+34)$, the estimation was not possible, since components show an inverted spectrum, which means we are looking at the self-absorbed part of the spectrum: in this case, the measured flux density is biased by the absorption, and does not reflect the real emission of the component.

We found values of $u_{\mathrm{min}}$ ranging from $10^{-7}$ to $10^{-5} \mathrm{erg} / \mathrm{cm}^{3}$, corresponding to $B_{\text {eq }}^{\prime}$ between 0.8 and $6.5 \mathrm{mG}$. In Sect. 4 we compare these results with values from literature. It is worth noting that some a priori assumptions have been made in this estimation. The value of $k$, the filling factor of the magnetic field $\phi$, and the depth of the source along the line of sight $d$ can have slightly different values from the ones used here, changing the results up to an order of magnitude.

\section{Discussion}

Radio-loud BAL QSOs were considered extremely rare sources before the advent of large surveys like the SDSS. After the release of SDSS DR4, it has been possible to select larger samples of BAL QSOs with a considerable emission also in the radio domain. The sample we studied here has been defined to have the largest possible number of sources with radio flux density high enough to allow a detailed study of the pc-scale structure.

In the literature, $32 \mathrm{BAL}$ QSOs have to date been observed with the VLBI imaging technique (Jiang \& Wang 2003; Kunert-Bajraszewska et al. 2007, 2010; Liu et al. 2008; Montenegro-Montes et al. 2008b; Gawroński \& Kunert-Bajraszewska 2011). With this work we add a further nine previously unobserved sources, improving the statistics concerning the pc-scale radio morphology. Only 8 BAL QSOs out of $32(\sim 25 \%)$ were found to be unresolved in earlier works, while the remaining objects show a variety of morphologies, including core-jet, quite symmetric, or complex structures. In some cases, a re-orientation or jet-precession scenario has been proposed (Kunert-Bajraszewska et al. 2007, 2010; Gawroński \& Kunert-Bajraszewska 2011) to explain the peculiar morphology. Doi et al. (2009) have observed another 22 BAL QSOs with the Optically ConnecTed Array for VLBI Exploration (OCTAVE), subarray of the Japanese VLBI Network, to detect pc-scale radio emission due to non-thermal jets. Their work does not provide maps, hence no morphological information comparable to the previous, but also in this case the results did not point to a single pole-on geometry for BAL QSOs.

Concerning our sample, four sources $(16 \%)$ out of the 25 in the whole sample $(0816+48,0849+27,1103+11$, and $1603+30)$ were already resolved at the intermediate angular resolution of our previous VLA observations (Bruni et al. 2012), resulting in linear sizes of $217,380,69$, and $17 \mathrm{kpc}$, respectively. The remaining $84 \%$ of the sample turned out to be unresolved, with a linear size below $20 \mathrm{kpc}$ in $90-95 \%$ of the cases. A previous 
G. Bruni et al.: The parsec-scale structure of radio-loud broad absorption line quasars

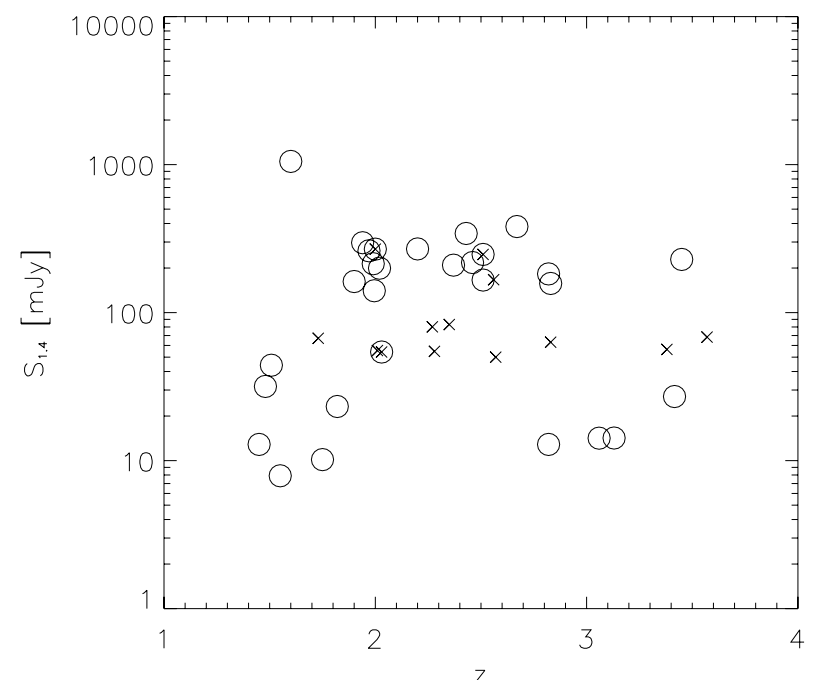

Fig. 4. Integrated flux density from the FIRST versus redshift for the 13 BAL QSOs with VLBI observations comprised in the Bruni et al. (2012) sample (from this paper or from Montenegro-Montes et al. $2008 \mathrm{~b}$ - crosses) and from the literature (circles). For source 0849+27, resolved at the FIRST resolution, we considered the flux density given by the sum of the single components.

VLBI study by our group (Montenegro-Montes et al. 2008b), based on a fainter sample of radio-loud BAL QSOs presented in Montenegro-Montes et al. (2008a), shows that on the parsec scale a variety of morphologies are found, implying different orientations. In that work a total of five sources were observed with the VLBA, resulting in two resolved sources (40\%). In this work we present pc-scale observations of eleven sources: nine of them present a resolved structure $(\sim 82 \%)$ without a predominant morphology. Both double sources with a radio spectrum consistent with being young (and implying jets oriented to large angles to the line of sight) and core-jet objects (with their axis quite aligned to the line of sight) have been found, reinforcing the idea that orientation is not the dominant factor in determining the properties of BAL QSOs. In our pc-scale images, three sources $(0756+37,1014+05$, and $1406+34)$ can account only for part of the VLA flux density measured at the same frequency. This missing flux density could be related to some emission with angular scales not sampled by the VLBI baselines (i.e. exceeding several tens of milliarcseconds, and therefore resolved out by the observations presented here) but contributing to the arcsecond scale measurement. Otherwise, such a difference may arise from intrinsic variability of the most compact components. All of these three sources were unresolved in the VLA maps, with linear size below $8 \mathrm{kpc}$.

If we also consider the sources $1159+01$ and $1624+37$, studied by Montenegro-Montes et al. 2008b, we end up with a total of 11 out of $13(\sim 85 \%)$ sources that appear resolved on the pc-scale. In $1159+01$ a central core was found, with two faint symmetric extensions and another two components toward SW, while in the case of $1624+37$ a core-jet structure was detected. This is consistent with the variety of morphologies we have found in the present work, and it suggests a variety of orientation with respect to the line of sight, in agreement with the results in Bruni et al. (2012).

These results, as a whole, agree with the findings available in the literature, both in terms of unresolved sources fraction and in possible morphologies (and orientations). Figure 4 shows the integrated flux density from the FIRST versus redshift for the 13 objects with VLBI observations in the sample from Bruni et al. (2012) and from the literature. Both redshift and flux density ranges are comparable to the literature, so we can safely include the results from this paper in the context of previous VLBI observations of BAL QSOs.

After a decade of VLBI observations, BAL QSOs do not seem to be predominantly unresolved, or to present any preferred morphology. All of them have been detected on the pc-scale. In some cases these sources can be compact, with a projected linear size below $20 \mathrm{kpc}$, or even extremely compact, $<1 \mathrm{kpc}$, but there are examples of extended sources (hundreds of kpc), like $0849+27$, presenting BAL features, or cases of BAL QSOs with traces of a diffuse emission, probably related to a low-frequency component remnant of an earlier active phase. Thus, the interpretation of BAL QSOs as young sources seems to be overly simplistic. On the other hand, the variety of jet axis orientations with respect to the line of sight we found does not allow us to explain this particular subset of QSOs as likely to be seen from a particular line of sight, as suggested by the orientation model (Elvis 2000). The outflows at the origin of the BAL features should thus be present at various orientations with respect to the jet axis, and in various phases of the QSO evolutionary track.

We determined the magnetic field intensity in the various emitting regions, under the conventional assumptions of pure synchrotron emission and minimum total energy (i.e. the equipartition field), and we found values of a few $\mathrm{mG}$. Dallacasa et al. (2002a,b) present VLBA, EVN and Merlin observations of a sample of compact steep-spectrum sources (CSS) drawn from the B3-VLA sample (Vigotti et al. 1989) and covering about the same radio luminosity range of the BAL QSOs presented here. They calculated the minimum energy density and the magnetic field strength, adopting the same assumptions and methodology, for radio components with sizes and flux densities comparable to the ones presented in this work. They found $u_{\text {min }}$ in the range between $10^{-8}$ and $10^{-7} \mathrm{erg} / \mathrm{cm}^{3}$, corresponding to $B_{\mathrm{eq}}^{\prime}<3 \mathrm{mG}$, comparable with our findings. This suggests that the radio-emitting plasma in radio-loud BAL QSOs has physical conditions similar to other (non-boosted) sources, while typical values for large-scale radio sources, such as FRI and II, spans from tens to hundreds of $\mu \mathrm{G}$ (Croston et al. 2005; Stawarz et al. 2005; Stawarz \& Kataoka 2005).

\section{Conclusions}

We have presented the results of EVN and VLBA observations of 11 among the brightest radio-loud BAL QSOs in our sample. A pc-scale imaging, together with a spectral index analysis of the components, was realized. We can summarize our conclusions as follows.

- Nine out of eleven sources $(82 \%)$ present a resolved structure, and various morphologies are visible: double, core-jet and symmetric structures have been found, and therefore different orientations can be inferred. The percentage of resolved sources is comparable to the general results from earlier works in the literature.

- The projected linear sizes of the sources presented here range from a few pc (upper limit for the unresolved sources) to several tens to several hundred pc. A few sources have additional radio emission on much larger scales, as is visible in the VLA observations, and therefore a typical radio size of BAL QSOs does not exist. These objects explore the same parameter space as the common radio sources (radio galaxies and radio quasars). In the original sample from 
Bruni et al. (2012), $\sim 80 \%$ of sources have linear sizes below $20 \mathrm{kpc}$, still indicating that, on the arcsec-scale, the majority of BAL QSOs remain unresolved.

The missing flux density between VLA and VLBI observations can suggest in some cases a diffuse emission that is too faint to be detected with these observations. In one case $(1406+34)$ this can be due to a low-frequency component, probably the remnant of an earlier radio activity of the AGN. A complex history can also be supposed for other sources, when a re-oriented jet seems to be at the origin of the complex morphology found.

- The variety of linear sizes and spectral characteristics found in these VLBI observations and from our previous work (Bruni et al. 2012) seems to exclude a simple explanation for all BAL QSOs as young compact objects. At the same time, given the variety of morphologies that can be found, a particular orientation is unlikely to be present: the outflows triggering BAL features are then likely to be present in both young and older QSOs, at various orientations with respect to the jet axis.

- A calculation of the minimum energy density and magnetic field strength shows similar values to the ones present in the literature, suggesting that no particular conditions are present in the radio-emitting regions of BAL QSOs.

Acknowledgements. We would like to thank M. Mahmud for kindly helping us with the preparation of the EVN schedules. Part of this work was supported by a grant of the Italian Programme for Research of Relevant National Interest (PRIN No. 18/2007, PI: Mack). The authors acknowledge financial support from the Spanish Ministerio de Ciencia e Innovación under project AYA2008-06311C02-02 and AYA2011-29517-C03-02. The European VLBI Network is a joint facility of European, Chinese, and other radio astronomy institutes funded by their national research councils. The National Radio Astronomy Observatory is a facility of the National Science Foundation operated under cooperative agreement by Associated Universities, Inc. This work made use of the Swinburne University of Technology software correlator, developed as part of the Australian Major National Research Facilities Programme and operated under licence.

\section{References}

Becker, R. H., White, R. L., \& Helfand, D. J. 1995, ApJ, 450, 559 Briggs, F. H., Turnsheck, D. A., \& Wolfe, M. 1984, ApJ, 287, 549 Brunetti, G., Setti, G., \& Comastri, A. 1997, A\&A, 325, 898

Bruni, G., Mack, K.-H., Salerno, E., et al. 2012, A\&A, 542, A13

Croston, J. H., Hardcastle, M. J., Harris, D. E., et al. 2005, ApJ, 626, 733

Dallacasa, D. 2003, PASA, 20, 79

Dallacasa, D., Tinti, S., Fanti, C., et al. 2002a, A\&A, 389, 115

Dallacasa, D., Fanti, C., Giacintucci, S., et al. 2002b, A\&A, 389, 126

de Bruyn, G., Miley, G., Rengelink, R., et al. 2000, VizieR On-line Data Catalog, $\mathrm{VIII} / 62$

Deller, A., Brisken, W. F., Phillips, C. J., et al. 2011, PASP, 123, 275

DiPompeo, M. A., Brotherton, M. S., De Breuck, C., et al. 2011, ApJ, 743, 1, 71

Doi, A., Kawaguchi, N., \& Kono, Y. 2009, PASJ, 61, 6, 1389

Elvis, M. 2000, ApJ, 545, 63

Gawroński, M. P., \& Kunert-Bajraszewska, M. 2011, PoS (X EVN Symp.), 037

Govoni, F., \& Feretti, L. 2004, IJMP, 13, 08, 1549

Ghosh, K. K., \& Punsly, B. 2007, ApJ, 661, 139

Hewett, P. C., \& Foltz, C. B. 2003, AJ, 125, 1784

Jiang, D. R., \& Wang, T. G. 2003, A\&A, 397, L13

Kunert-Bajraszewska, M., \& Marecki, A. 2007, A\&A, 469, 437

Kunert-Bajraszewska, M., Janiuk, A., Gawroński, M. P. et al. 2010, ApJ, 718 , 1345

Liu, Y., Jiang, D. R., Wang, T. G., et al. 2008, MNRAS, 391, 246

Montenegro-Montes, F. M., Mack, K.-H., Vigotti, M., et al. 2008a, MNRAS, 388,1853

Montenegro-Montes, F. M., Mack, K.-H., Benn, C., et al. 2008b, POS (IX EVN Symp.), 019

O'Dea, C. P., \& Baum, S. A. 1997, AJ, 113, 148

Punsly, B. 1999a, ApJ, 527, 609

Punsly, B. 1999b, ApJ, 527, 624

Sanders, D. B. 2002, ASP Conf. Ser., 284, 411

Schneider, D. P., Hall, P. B., Richards, G. T., et al. 2007, AJ, 134, 102

Shankar, F., Dai, X., \& Sivakoff, G. R. 2008, ApJ, 687, 859

Stawarz, L., \& Kataoka, J. 2005, Proc. 22nd Texas Symposium on Relativistic Astrophysics at Stanford, California, 471

Stawarz, L., Siemiginowska, A., Ostrowski, M., \& Sikora, M. 2005, Proc. 22nd Texas Symposium on Relativistic Astrophysics at Stanford, California, 475

Vigotti, M., Grueff, G., Perley, H., et al. 1989, AJ, 98, 419

White, R. L., Becker, R. H., Gregg, M. D., et al. 1997, BAAS, 29, 1373

Zhou, H., Wang, T., Wang, H., et al. 2006, ApJ, 639, 716 
G. Bruni et al.: The parsec-scale structure of radio-loud broad absorption line quasars

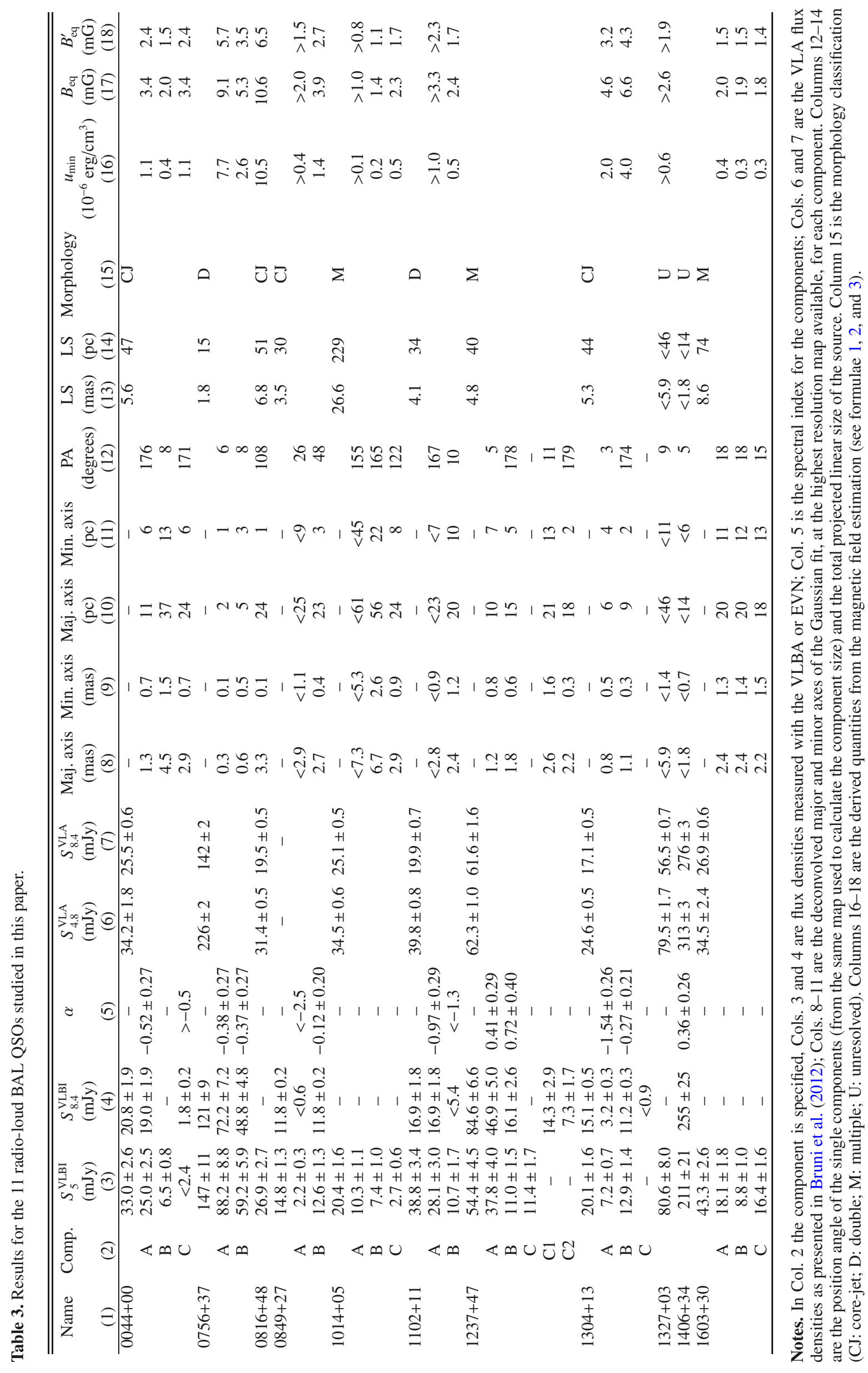

A94, page 11 of 11 